\title{
AN ADVANCED HOME ELDERCARE SERVICE
}

\author{
Tarik Taleb \\ Graduate School of Information Sciences, Tohoku University, Sendai, Japan \\ talebtarik@ieee.org \\ Dario Bottazzi \\ Department of Electronics, Computer Science and System Engineering, University of Bologna, Bologna, Italy \\ dario.bottazzi@unibo.it \\ Hammadi Nait-Charif \\ Bournemouth Media School, Bournemouth University, Bournemouth, UK \\ hncharif@bournemouth.ac.uk
}

Keywords: ubiquitous assistance, pervasive computing, healthcare network, and elderly

\begin{abstract}
With the increase of welfare cost all over the developed world, there is a need to resort to new technologies that could help reduce this enormous cost and provide some quality eldercare services. This paper presents a middleware-level solution that integrates monitoring and emergency detection solutions with networking solutions. The proposed system enables efficient integration between a variety of sensors and actuators deployed at home for emergency detection and provides a framework for creating and managing rescue teams willing to assist elders in case of emergency situations. A prototype of the proposed system was designed and implemented. Results were obtained from both computer simulations and a real-network testbed. These results show that the proposed system can help overcome some of the current problems and help reduce the enormous cost of eldercare service.
\end{abstract}

\section{INTRODUCTION}

The structure of world population growth is changing. It is also growing older, particularly in developed world societies. On another hand, due to many geographic (e.g., earthquakes) and socio-political factors (e.g., wars), a non-negligible fraction of the world population is suffering from different impairments (e.g., vision, hearing, cognitive, etc) that deprive people from independently performing basic activities (e.g., social interactions, household, and self-care). This growing percentage of elderly people in the society and the increasing number of patients in need of home assistance put welfare systems under relevant stress.

To cope with this emerging issue, there is need for novel forms of cost-effective welfare services and technologies capable of accommodating the increasing number of people in need of assistance. In this vein, the US National Center on Medical Rehabilitation Research (NCMRR) has defined five main directions for research related to the provision of assistance to people in need of help. These directions pertain to cellular (e.g., genetic abnormalities), organ (e.g., cardiovascular pathology), action (e.g., paralysis), task- role (e.g., Alzheimer), and social limitations (e.g., isolation, depression, and memory impairment) (Mann, 2004). The two last areas form the main research focus in the NCMRR model. Indeed, there is a large research body working on solutions at the task-role level and addressing the social barriers. The devised solutions aim at improving the quality of life of the elderly, enabling their autonomy in their daily activities, and reducing their dependency on caregivers, families, and friends. This is with every intention to render their living environments more socio-emotional. Unlike the cellular area whereby the solutions are more or less medical curing-based, the focus of all other NCMRR areas is on the development of technological solutions.

Indeed, a relatively new and fast growing trend in the world of technology is the use of telecommunication technologies and pervasive computing as a tool in the practice of medicine. The research work outlined in this paper aims at designing a healthcare/welfare service that enhances the daily life independence of elderly in indoor environments. The main motivation behind this research work emerges from the conviction that recent and ongoing advances in wireless technologies, sensors, wearable devices, and com- 
puter vision techniques, if integrated well, can offer unique chances to improve the quality of life and provide vital assistance to elderly.

We therefore intend to design a wellness system based on an efficient integration of pervasive computing technologies and computer vision techniques to provide safety and quality of life to elderly and patients in need of help. In this research work, wearable devices, sensors and computer vision techniques are used for motion tracking, constant monitoring of the patient's behavior, and based on that, detection of behavioral abnormalities. On the other hand, telecommunication technologies are used to form groups of volunteers and caregivers willing to help in case of an emergency event, and thus provide elders with a sense of safety. Their usage can be also extended to forming communities of elders with common interest and fostering social interactions among them (Bottazzi et al., 2007). For the sake of better customized eldercare customized solutions, it would be worth focusing the work on one particular pathology, e.g., Alzheimer, cardio-pathology. However, in this research work, we present the general concept of our envisioned eldercare service which can be easily tailored to any specific pathology taking into account different factors such as clinical conditions of the pathology and cultural profile of the patient.

Intuitively, the main advantage behind this research work consists in the reduction of the social and economic overhead of the hospitalization of elderly and patients. Indeed, such a system will be deployed where seniors live, will provide them with more autonomy in carrying out their daily activities (e.g., reminding Alzheimer patients of routing activities), and will assure them immediate assistance (from either passing by volunteers or nursing experts) in case of emergency events. This shall postpone or completely avoid the transfer of elderly into nursing homes and shall eventually allow health welfare of elderly to be decentralized from hospital systems.

The remainder of this paper is organized as follows. Section II provides a survey on a number of systems recently devised for ubiquitous eldercare and discusses the advantages and pitfalls of each of them. Section III describes the key components of the envisioned home healthcare service. The major operations of the proposed eldercare system are described in detail in Section IV. The performance of the proposed eldercare service architecture is evaluated in Section V. The paper concludes in Section VI with a summary recapping the main advantages and achievements of the proposed service.

\section{RELATED WORK}

Ubiquitous eldercare has been gaining a great momentum in the last few years at both industry and academia levels. Different solutions have been proposed. Depending on their design goals, these solutions can be categorized into two groups, namely ubiquitous assistance and ubiquitous care networking support.

In the first category, there has been a plethora of research work addressing various aspects of telemedicine (Soomro and Cavalcanti, 2007). The scope of these researches ranges from standardization activities for telemedicine deployment (Choi et al., 2006), privacy and security solutions (Xiao et al., 2006) to realization of a system (Hodgins et al., 2008; Poon et al., 2006; Garawi et al., 2006). The main concerns of these solutions consist in assisting elders in their routine life activities, constant monitoring of their health conditions, and prompt alerting in emergency events. In (Marquis-Faulkes et al., 2003), a computer-vision based system is proposed to support people with severe vision impairments. To secure a safe navigation of a particular environment, the system generates alert messages, via a speech output interface, whenever a change occurs to the layout of the environment. This makes people, with vision deficiency and living in that environment, aware of the occurring changes. In (Mihailidis et al., 2004), artificial intelligence learning and planning techniques are used to define proper steps of basic activities of daily living (e.g., hand-washing). The resultant system provides visual or verbal instructions to a person with dementia on how to perform a particular daily living activity. The system consists of three modules; a tracking module which uses computer vision to monitor the actions of the user by determining the spatial coordinates of the person's body and hands within the environment. Once these coordinates have been determined, a planning module determines what step the user is completing and whether the step being completed is correct. If the system detects that the user has made an error, such as completing a step out of sequence or missing a step altogether, the prompting module selects and plays a prompt message. In (Pollack et al., 2003), a cognitive orthotic system, called auto-minder, is proposed. The system models the daily plans of an individual and decides on when and where to remind the person of the execution of those plans. The developed techniques are deployed on a mobile robot, as part of the Nursebot project Initiative on Personal Robotic Assistance of the Elderly. Benny et al., in their UbiSense system, used embedded smart vision techniques to de- 
tect changes in posture, gait and activities. In addition to monitoring normal daily activities and detecting potential adverse events such as falls, the system aims to capture signs of deterioration of the patients by analyzing subtle changes in posture and gait (Lo et al., 2005). In their later work, they used image sensing and vision-based reasoning to verify and further analyze events reported by other sensors such as Accelerometer for fall detection (Tabar et al., 2006). The Honeywell Laboratories' Independent Life Style Assistant(ILSA) is another notable example of integrated smart environments which aim at ubiquitous assistance (Haigh et al., 2004). In the ILSA system, multiple JADE agents are deployed. The agents support data monitoring via home-installed sensors. The collected data are aggregated and processed to make adequate response planning and machine learning. UbiMedic (Mola et al., 2006) and Code Blue (Pesto et al., 2008) are other notable agent-based smart home assistance systems. A general observation about the different industrial and academic proposals of ubiquitous assistance is that they focus on only specific tasks, and thus provide only a subset of the support functions needed for eldercare assistance. A thorough discussion on major ubiquitous assistance solutions, their requirements and challenges can be found in (Bellavista et al., 2007).

The ubiquitous care networking area is still in its infancy, with only a few solution proposals. The primary objective of ubiquitous care networking solutions is to promote social interactions of elders with their surroundings and to coordinate between the different actors involved in a ubiquitous eldercare event. In (Bottazzi et al., 2006), a context-aware middleware solution, dubbed Allocation and Group Aware Pervasive Environment (AGAPE), is proposed for the creation and management of ad-hoc assistance teams to provide outdoor emergency assistance to senior citizens in need of immediate help.

To conclude, different solutions have been devised in the recent literature for both ubiquitous assistance and ubiquitous care networking support. To the best knowledge of the authors, no solution in the literature has integrated the two types of ubiquitous eldercare into one single solution. This challenging task underpins the focus of the research work outlined in this paper.

\section{ARCHITECTURE DESCRIPTION}

Fig. 1 depicts the envisioned architecture and its major components. The figure portrays the case of a

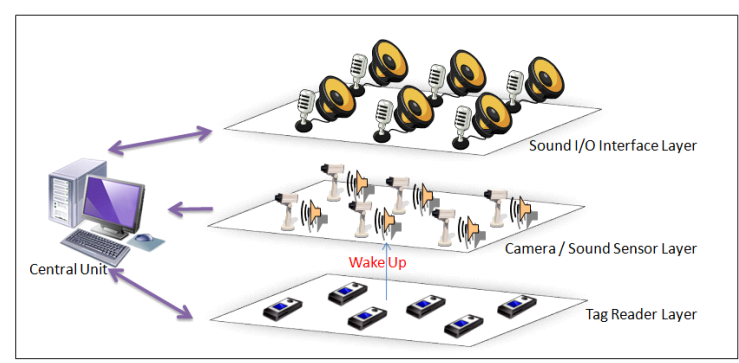

Figure 2: Risk-mining of inputs from multiple sensor layers.

smart indoor environment (e.g., living house) embedding a variety of wireless devices, namely RFID (Radio Frequency Identification) readers, advanced camera sensors, sound sensors, voice input/output interfaces, and a central unit (e.g., PC). The house is also equipped with a smart door lock with a passwordopening function. The central unit is connected to a surveillance center via the Internet and installs IBM via voice in order to provide elders with a speech based interface.

A RFID tag is adhered to the targeted senior or a device that is frequently used by the person. Costeffective RFID readers are intelligently placed over the house in a way that they provide complete coverage over the entire house. To avoid reader-to-tag interferences, two adjacent readers are placed in a way that the difference between their signal strengths is less than the tag's tolerance margin. By functioning as the Reader Network Controller (RNC), the central unit coordinates among the multiple readers using the EPCglobal Low-Level Reader Protocol (LLRP).

In the considered architecture, all devices are logically located in three layers based on the order of their inputs, as portrayed in Fig. 2. RFID readers are placed in the first layer. They are used to detect the senior when he gets nearby. Their feedback is sent to the central unit. The latter uses this information to trigger camera and sound sensors that are covering the area (i.e., room) where the person is currently located. Other cameras and sound sensors covering other rooms are kept off. This is for the sake of efficient usage of energy (i.e., electricity). It also permits the central unit to analyze only images obtained from a single camera, thus reducing both home network overhead and computational load that may be induced by computer vision techniques. It should be noted that this operation can be also performed using motion sensors. However, in case the senior is sharing the house with other members of the family, the use of motion sensors is not efficient as they cannot distinguish the senior from the other family members. 


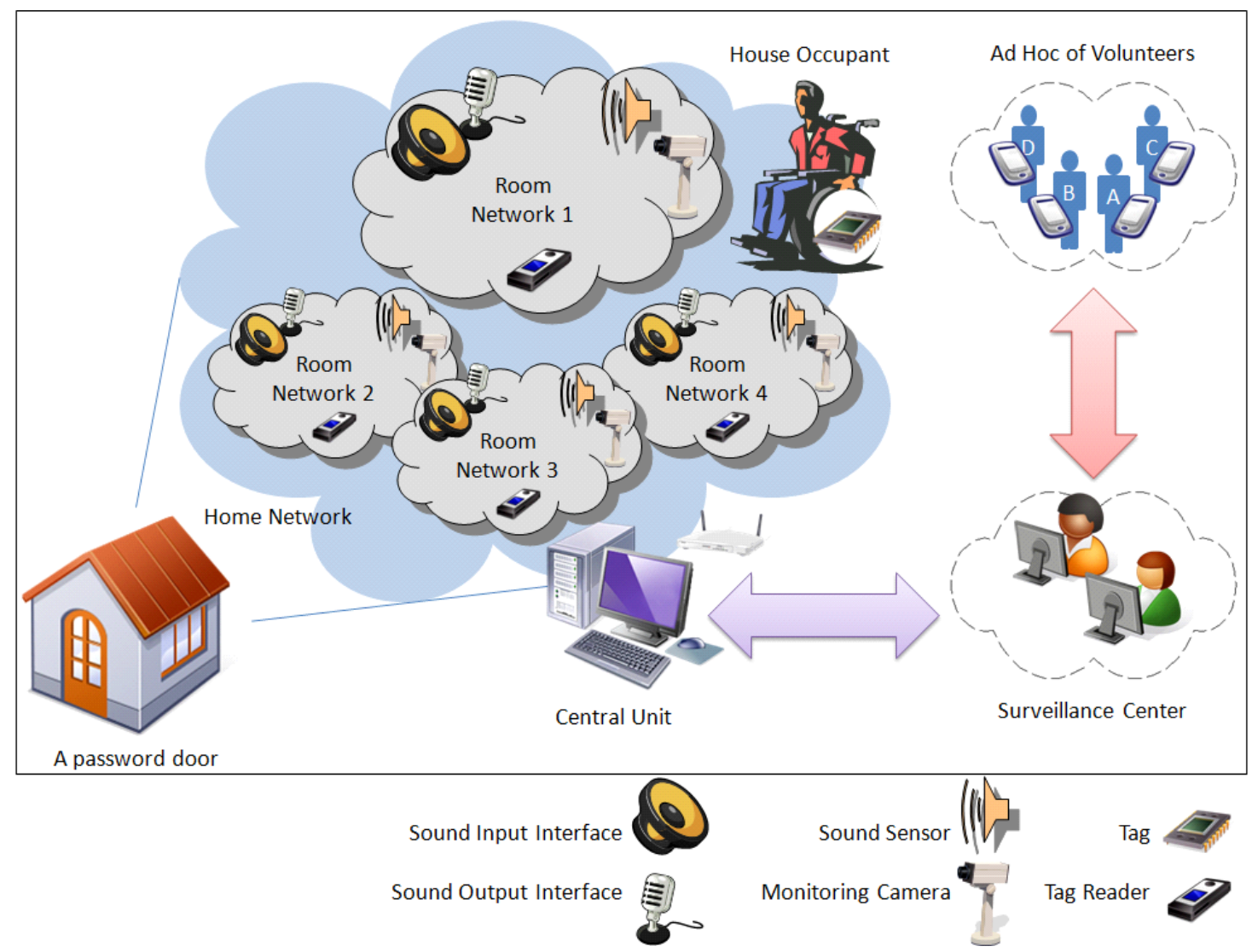

Figure 1: A smart living house with a set of intelligent devices, each with a different task.

Advanced camera sensors are used for human motion monitoring (Nait-Charif and McKenna, 2003). Their feedback is transmitted to the central unit. The central unit uses this feedback to track the person and to keep records of his motion. As will be explained in the next section, the central unit uses also feedback from the camera sensors to make decision on whether an anomaly occurred in the behavior of the senior. For this purpose, the central unit applies a set of algorithms to judge the conditions of the senior (whether he is in a normal or an emergency situation) (McKenna and Nait-Charif, 2004). Sound sensors are used to enhance the anomaly detection (e.g., in case the monitored senior falls down or screams for help). For higher accuracy in capturing the elder's behavior, the central unit needs full visibility of the elder's context, including special layout of the objects in his living environment. Such context awareness is possible by tagging items that are frequently used by the senior (e.g., TV set, Sofa). Whenever a change occurs in the layout of the living environment, updates of the layout is possible via an efficient integration between the inputs from the tag readers and the cameras.
Upon the detection of an anomaly, both voice input and output interfaces are turned on. The central unit first confirms with the senior if there is need for any kind of assistance. In case this is confirmed by the senior or there is no response from the senior within a predefined timeout, a virtual connection is set between the central unit and the health-care service administrator (i.e., surveillance center). The audio and video captured from both the camera and sound sensors, respectively, during the time of the emergency event are sent to the surveillance center via the Internet. As will be explained in the next section, the surveillance center follows a number of steps to form groups of volunteers that may provide the necessary assistance to the senior. The service administrator will provide the selected volunteers with the password to access the house of the senior. Major operations of the proposed eldercare system are portrayed in Fig. 3. 


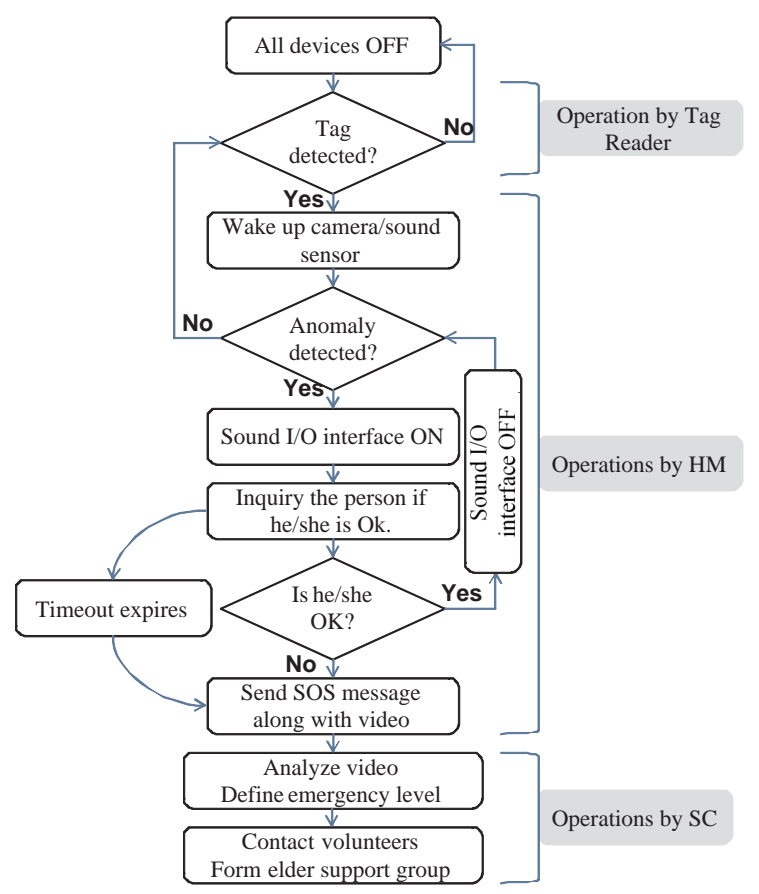

Figure 3: Major operations of the proposed eldercare system.

\section{PROPOSED ELDERCARE SYSTEM}

\subsection{Computer Vision based Monitoring and Anomaly Detection}

At the start of the system, the central unit develops a normal behavior model of the elder (during the learning or profiling phase). This model can be developed when the elder is supervised by a nurse for a short period of time.

While receiving feedback from cameras and sound sensors, the central unit compares the current behavior with the normal behavior using computer vision techniques to judge the normalcy or the anomaly of the instance. A set of predefined rules can be used for setting up an alarm in case of anomaly.

While computer vision-based anomaly detection is not the focus of the research work outlined in this paper, there has been a wide library of research work in the literature on computer vision techniques (McKenna and Nait-Charif, 2004; Cucchiara et al., 2007). These techniques are used to track the occupant in his home environment and an AI system learns the daily life pattern in order to detect any abnormalities such as falls or unusual activity/inactivity patterns that can be attributed to health problem. Moreover,

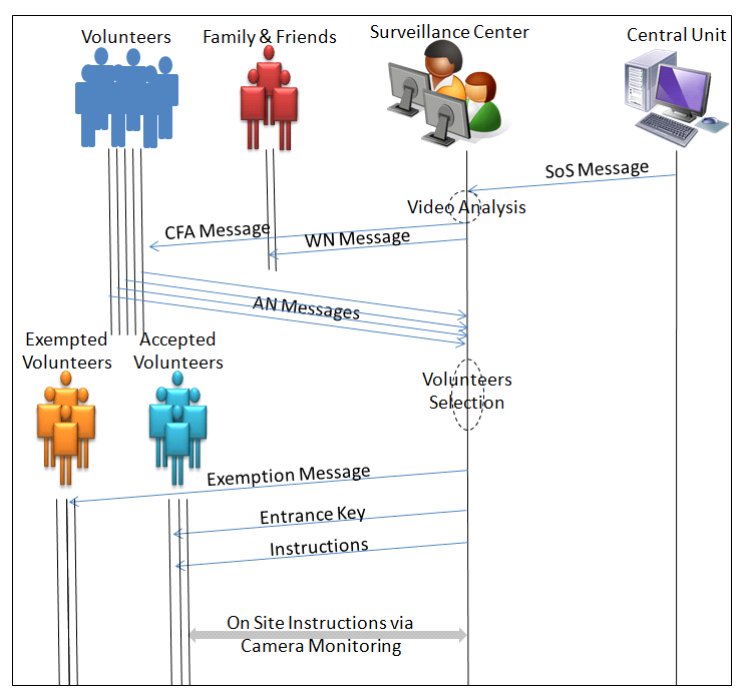

Figure 4: Sequence of signaling messages exchange.

computer vision can be used to collect more in-depth information in order for the system to build more accurate models of the user environment and help the agents at the surveillance center to look retrospectively to events leading to any unusual event such as a fall. In (McKenna and Nait-Charif, 2004), a system is developed for automatically tracking a single occupant in his home environment (sitting room) and annotating his activities and detecting any abnormal inactivity which might be a fall. In this system, the occupant is tracked for an extended period of time for the central unit to learn the usual entry/exit and inactivity zones in the sitting room. Once the learning is done, computer vision techniques are used to detect any unusual inactivity which can indicate a matter of concern such as a fall and can be used as part of an alarm to alert the surveillance center. In this system, an inactivity was detected with a delay $\tau_{d}=1.6 \mathrm{sec}$ onds.

\subsection{Call for Help upon Risk Detection \& Clustering of Ad-Hoc Volunteers Willing to Help}

Upon detection of any abnormalities such as falls, faintness, or unusual activity/inactivity patterns that can be attributed to health problem, the central unit follows the steps depicted in Fig. 3. First of all, the unit plays a prompt message via the voice output interface inquiring the person if he is alright or, indeed, in need of assistance. Awaiting a response from the person, the system sets up a timeout. In case the person confirms that he is in need of help or the timeout 
expires, the system judges the event as an emergency situation and an alert message is sent to the surveillance center in the form of a SoS message. Intuitively, such confirmation or timeout aims at reducing false alerts to the surveillance center.

The alert message will be accompanied with the capture video so the health-care service personnel may look retrospectively at the moment just before the event occurrence to analyze the cause of the event and to decide whether the person is in an emergency situation. Based on the capture video and the clinical profile of the elder, the agent at the surveillance center also defines the emergency level and type, its causes, and the kid of help the person may be in need of.

To ensure a prompt assistance to the person, the service administrator sends two types of messages: i) a Warning Notification (WN) message to family members, friends and relatives living in the immediate surroundings of the person, and a Call For Assistance (CFA) message broadcast to a database of volunteers (e.g., passers-by, neighborhood community representative and paid help, such as professional caregivers, doctors, pharmacists, etc). To avoid flooding the whole network with CFA messages, CFA messages are broadcast only over a particular locality composed of a limited number of access points covering an area that forms a circle with the residence of the senior at its center and a radius $r$. This locality concept both mitigates the complexity of the group formation problem as it limits the group management scope and reduces responders' intervention time.

CFA messages include information such as personal information of the senior (e.g., age, gender, etc), the postal address of his residence, his physical and cognitive characteristics, the kind of assistance he is in need of, along with additional information (if available) describing the current conditions of the elder (e.g., pulse). In response to the CFA message, volunteers willing to help send back an Acceptance Notification (AN) message to the surveillance center. These reply messages contain personal information of the volunteers (e.g., name), their current location, and the estimated time it may take them to get to the location of the person in need of help. Fig. 4 depicts the sequence of signaling messages exchanged between the major participants of the proposed eldercare service architecture.

In case of multiple replies from multiple volunteers, the surveillance center sorts out the most adequate ones based on their geographical proximity to the residence of the person, and some other information already available in the database of volunteers, such as their medical expertise, their history record and skills in providing such assistance, and the trust the surveillance center associates with them. The sourcing of adequate volunteers from a group of repliers prevents bystander apathy effect, which may inhibit responders from providing assistance to the elder. As will be explained later, it is based on the multi-attribute decision making (MADM) theory.

Once the volunteers are sorted out, the surveillance center notifies the selected ones of the password to access the door of the person's residence and the other volunteers are simply exempted and thanked for their eagerness to help (Fig. 5). At the same time, the surveillance center provides the selected volunteers with instructions on how to assist the person. Instructions can be either in a text or voice mode. They consist of "what-to-do" and "how-to-do" lists pertaining to tasks that need to be performed. Each task entry in the "what-to-do" list is associated with an entry into the "how-to-do" list that briefly instructs users, untrained or unfamiliar with the system, on how to perform the corresponding tasks. Once the volunteers enter the residence of the person in need of help, the agent at the surveillance center keeps monitoring them using the cameras available at the residence of the person and providing them with further instructions whenever necessary.

It should be emphasized here that while it is possible to consider a self-functioning approach where upon risk detection the central unit wirelessly broadcasts CFP messages to an ad-hoc of passers-by, with no involvement of a central surveillance center similar in spirit to the outdoor emergency assistance system proposed in (Bottazzi et al., 2006), such an approach gives rise to two major issues, mainly safety and false alerts. The first issue is highly important as we are aiming at indoor environments and the second is due to the fact that computer vision still has limitations in accurately capturing one's activities and fully understanding his behavior from a video sequence, despite of the wide library of research in this area.

\subsection{Volunteers Selection Mechanism}

In this section, we describe the philosophy behind the selection of adequate volunteers to cope with a particular emergency situation. As stated earlier, the volunteer selection mechanism is based on the multiattribute decision making theory. Indeed, in the proposed system, the surveillance center maintains profiles of each volunteer; already subscribed to the service. For each subscriber $S_{k}$, a set of attributes $\left\{X_{k, j}, j \in\{1,2, \cdots, \ell\}\right.$ is associated. The attributes represent $i$ ) the expertise and skills of volunteers, $i i$ ) their history record in providing assistance, and iii) the trust level the surveillance center associates with 


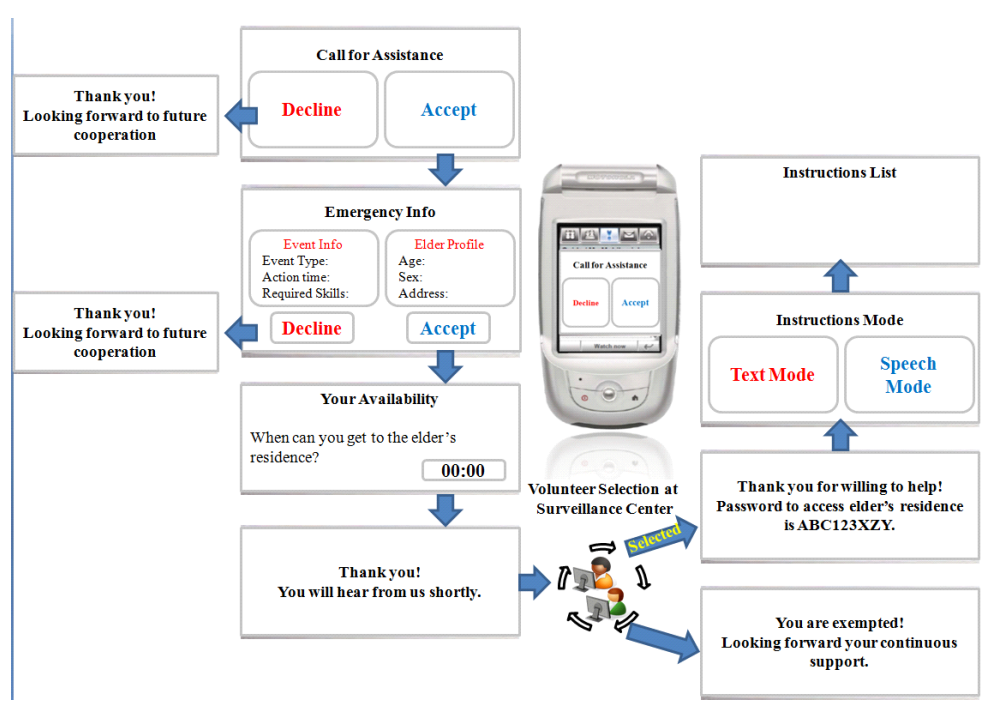

Figure 5: A prototype of application user interface.

them. It should be noted that these attributes are constantly updated and maintained by the surveillance center.

In the proposed system, we assume that there are $M$ emergency levels defined a priori at the surveillance center. For each emergency level $\mathfrak{I}_{i}(i \in\{1,2, \cdots, M\})$ and each attribute $X_{j}(j \in$ $\{1,2, \cdots, \ell\}$ ), the surveillance center defines a weight $\omega_{i, j}$. Additionally, with each emergency level $\mathfrak{I}_{i}$, three parameters are associated: $i$ ) $\theta_{\alpha . i}$ defining the minimum time within which assistance should be provided to the senior, $i i) \tau_{i}$ defining the maximum time (i.e., timeout) the surveillance center should wait for to receive AN messages from volunteers, and iii) $\gamma_{i}$ defining a threshold for selecting volunteers. It should be noted that these three parameters should be carefully set by the surveillance center. For example, in case of a life-threatening event (e.g., heart attack), both action time $\Theta_{\alpha . i}$ and waiting timeout $\tau_{i}$ should be set to small values. In case of a bone fracture due to a fall, expertise and skills become more important so the system can set $\gamma$ to high value with focus on the skills-related attribute. Action time $\theta_{\alpha}$ and timeout $\tau$ can be set to relatively high values.

Upon receiving a SOS message, the agent in charge (at the surveillance center) first defines the corresponding emergency level based on $i$ ) the event type determinable from the capture video (e.g., fall, faintness, heart-attack) and ii) the profile of the senior (e.g., physical and cognitive characteristics). Let $\mathfrak{I}_{m}$ denote the selected emergency level. For a timeout $\tau_{m}$, the agent waits for responses from volunteers. Once the system receives enough replies or the time- out $\tau_{m}$ expires, the system sorts out the replying volunteers based on information available in their AN messages, such as their physical proximity and availability, using the action time $\Theta_{\alpha . m}$. Indeed, as explained earlier, a volunteer $S_{k}$ notifies the surveillance center of the time he may need to reach the residence of the senior, should he be selected for the rescue task. Let $\Delta_{a . k}$ denote the availability of volunteer $S_{k}$ in terms of time. Using the action time $\Theta_{\alpha . m}$ of the emergency level, volunteers with time availability $\Delta_{a . k}$ satisfying the following inequality are selected.

$$
\Delta_{a . k} \leq\left\{\Theta_{\alpha . m}-\theta_{d}-\theta_{\text {SoS }}-\theta_{H}-\theta_{E}-\theta_{\text {select }}\right\}
$$

where $\theta_{d}$ refers to the time elapsed since the actual occurrence of the event till its detection by the central unit. $\theta_{S o S}$ denotes the time required to send SoS message and the capture video to the surveillance center. $\theta_{H}$ refers to the time required by the agent to analyze the video and to determine the emergency level. $\theta_{E}$ denotes the time required to exchange signaling messages (e.g., CFA, WN, AN) between volunteers and the surveillance center. $\theta_{\text {select }}$ denotes the time required by the surveillance center to run the volunteers selection algorithm.

At the second stage, the surveillance center sorts (Out of the selected volunteers) those with attributes satisfying the following condition.

$$
\sum_{n=1}^{\ell} X_{k, n} \cdot \omega_{m, n} \geq \gamma_{m}
$$

In case the number of volunteers selected at the second phase are numerous, to avoid bystander apathy effect only an adequate number of volunteers 
are requested to assist and are provided with password to the gate of the senior's residence along with instructions corresponding to the determined emergency level $\mathfrak{I}_{m}$.

\section{PERFORMANCE EVALUATION}

Our deployment settings are as shown in Fig. 1. The used cameras are standard digital video cameras operating at 30 frames per second, ceiling-mounted, with vertically-oriented optical axes, fitted with wideangle lenses. For monitoring the behavior of elders affected with severe vision impairments, the anomaly detection algorithm proposed in (McKenna and NaitCharif, 2004) is used. A Pentium-based PC running Gentoo Linux, J2SE 1.5 covering the central unit role was also deployed in the apartment. In particular, the system was appropriately configured and enabled to gather sensing information from the available sensors, i.e., RFID readers, sound sensors and cameras. In our testbed, we also deployed a PC running Gentoo Linux, J2SE 1.5 playing the surveillance center role. In addition, by exploiting the wireless Wi-Fi infrastructure deployed in our campus in University of Bologna, we also defined several localities. In our prototype, each network cell defines a locality. In each locality, we have also deployed a server, running Gentoo Linux, J2SE 1.5, managing among all terminals currently located in the locality. Finally, we also setup several wireless-enabled iPAQ PDAs, acting as user terminals for responders. Each device installs Personal Java, J/LMS, and client-side components. On top of the terminals, we also implemented an application prototype that provides available helpers with alert messages when an emergency situation occurs. Indeed, the application continuously makes the aware of the emergency notification by emitting a sound signal with an increasing volume till the user accepts or declines the request.

We have tested the system's responsiveness, defined as the amount of time required for forming an elder support group, in scenarios simulated with NS2 and consisting of a number of devices randomly deployed over the same locality, i.e., the same wireless LAN. To investigate the functionality of the system under different network conditions, we range the number of responders from 2 to 100 . Our simulation settings consider IEEE 802.11 networks with 10 Mbps transmission rate, random waypoint mobility pattern and speeds ranging from 1 to $3 \mathrm{~m} / \mathrm{s}$. For the sake of simplicity, users are assumed to respond immediately to CFA messages; time needed for a user to detect alerting messages is set to zero. Admittedly, in real-life scenarios, this time may dominate the overall group formation time. The simulations demonstrate that the system's responsiveness is, on average, of few seconds and tend to degrade to few tens seconds when more than 60 LRs are available. Responsiveness degrades due to increase in packet losses, due in turn to wireless network characteristics (e.g., limited bandwidth and channel error rates), in addition to a minimal contribution from the computational load that comes with fetching the profiles of users and with the users selection algorithm.

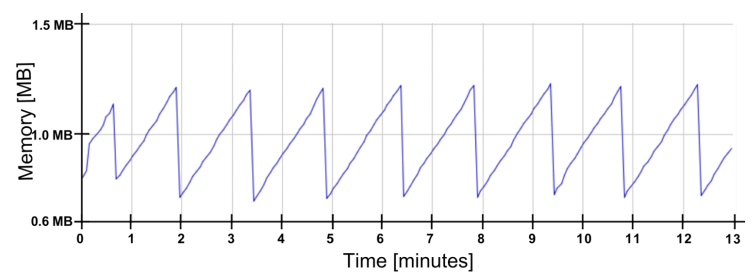

(a) Heap memory.

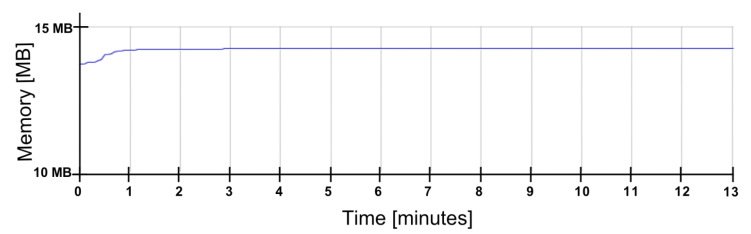

(b) Non-heap memory.

Figure 6: Memory use on responder devices.

Fig. 6 depicts the memory requirements as seen from a single responder device. The figure shows the overall memory requirements over time. In our experiments, we tracked memory use for a responder device in a locality where three other responders were available. The experiment lasted for a duration of 13 minutes. All data were obtained by exploiting the JConsol profiling tool. From Fig. 6-a, the total amount of the used heap memory varies between 0.7 $\mathrm{MB}$ and $1.3 \mathrm{MB}$, with an average value of about 0.9 MB. In addition, non-heap memory including data, code and stack reaches a stable value of about $14 \mathrm{MB}$ (Fig. 6-b). From these results, it can be concluded that the system's group management support can be easily installed over a PDA.

The system's overall responsiveness, defined as the time elapsed since the emergency occurrence till the arrival of the first helper to the residence of the senior in need of assistance, is an important metric for the system performance evaluation. However, it is highly difficult to exactly determine it. This is mainly due to the fact that the system responsiveness depends on unpredictable human factors, such as the behavior of the local responders (e.g., promptness in reacting to 
CFA messages) and their availability in terms of time which hinges, in turn, on their daily commitments. One way to cope with this challenge is to have the surveillance center form a statistical profile over time on the number of volunteers that visit its area of service and keep history track of the responsiveness of volunteers in terms of their reaction to CFA messages. Based on this statistical profile and history track, the surveillance center can define a process for the arrival of AN messages from volunteers, based on which it can make an estimate of the system's overall responsiveness to cope with a particular emergency situation occurring at a particular time in a particular locality.

\section{CONCLUDING REMARKS}

A prototype system has been developed to help elders at home. The proposed system is expected to also help reduce the cost incurred by the current eldercare service. The system is made of sensors and actuators that are connected to a central unit, acting as home network manager. The system is able of gathering and aggregating row information from sensing sources and detecting possible dangerous situations based on computer vision. A prototype user interface was also developed for responders' PDAs. The performance of the proposed system was evaluated based on both computer simulations and real-network experiments. The results were obtained in terms of battery consumption and memory use at helpers' terminals. Once fully implemented and adopted, we expect that the proposed system will have a great impact in improving eldercare services all over the world.

\section{REFERENCES}

Bellavista, P., Bottazzi, D., Corradi, A., and Montanari, R. (2007). Challenges, opportunities and solutions for ubiquitous eldercare, chapter Challenges, Opportunities, and Solutions for Ubiquitous Eldercare. Idea Group Inc.

Bottazzi, D., Corradi, A., and Montanari, R. (2006). Context-aware middleware solutions for anytime and anywhere emergency assistance to elderly people. IEEE Commun. Mag., 44(4):82-90.

Bottazzi, D., Montanari, R., and Toninelli, A. (2007). Context-aware middleware for anytime, anywhere social networks. IEEE Intelligent Systems Mag., 22(5):23-32.

Choi, Y. B., Krause, J. S., Seo, H., Capitan, K. E., and Chung, K. (2006). Telemedicine in the usa: Standardization through information management and technical applications. IEEE Commun. Mag., 44(4):41-48.
Cucchiara, R., Prati, A., and Vezzani, R. (2007). A multicamera vision system for fall detection and alarm generation. Expert Systems journal, 24(5):334-345.

Garawi, S., Istepanian, R. S. H., and Abu-Rgheff, M. A. (2006). $\quad 3 \mathrm{~g}$ wireless communications for mobile robotic tele-ultrasonography systems. IEEE Commun. Mag., 44(4):91-96.

Haigh, K. Z., Kiff, L. M., Myers, J., Guralnik, V., Geib, C. W., Phelps, J., and Wagner, T. (2004). The independent lifestyle assistant (i.l.s.a.): Ai lessons learned. In Proc. 16th Innovative Applications of Artificial Intelligence Conf. (IAAI'04). San Jose, CA, USA.

Hodgins, D., Bertsch, A., Post, N., Frischholz, M., Volckaerts, B., Spensley, J., Wasikiewicz, J., Higgins, H., von Stetten, F., and Kenney, L. (2008). Healthy aims: Developing new medical implants and diagnostic equipment. IEEE Pervasive Computing Mag., 7(1):14-21.

Lo, B., Wang, J., and Yang, G. (2005). From imaging networks to behavior profiling: Ubiquitous sensing for managed homecare of the. elderly. In Proc. 3rd Int'l Conf. on Pervasive Computing (PerCom 2005). Munich, Germany, IEEE Press.

Mann, W. C. (2004). The aging population and its needs. IEEE Pervasive Computing Mag., 3(2):12-14.

Marquis-Faulkes, F., McKenna, S., Gregor, P., and Newell, A. (2003). Scenario-based drama as a tool for investigating user requirements with application to home monitoring for elderly people. In Proc. 10th Int'l Conf. on Human-Computer Interaction (HCI'03). Crete, Greece, Crete University Press.

McKenna, S. J. and Nait-Charif, H. (2004). Summarising contextual activity and detecting unusual inactivity in a supportive home environment. Pattern Analysis and Applications, 7(4):386-401.

Mihailidis, A., Carmichael, B., and Boger, J. (2004). The use of computer vision in an intelligent environment to support aging-in-place, safety, and independence in the home. IEEE Trans. Information Technology in Biomedicine, 8(3):238-247.

Mola, F., Cabri, G., Muratori, N., Quitadamo, R., and Zambonelli, F. (2006). The ubimedic framework to support medical emergencies by ubiquitous computing. Int'l Trans. Systems Science \& Applications, 1(1):15-26.

Nait-Charif, H. and McKenna, S. J. (2003). Head tracking and action recognition in a smart meeting room. In 4th IEEE Int'l Workshop on Performance Evaluation of Tracking and Surveillance, pages 24-31. Graz, Austria, IEEE Press.

Pesto, C., Selavo, L., Chen, Y., Ko, J., Lim, J., Terzis, A., Watt, A., Jeng, J., Chen, B., Lorincz, K., and Welsh, M. (2008). Wireless medical sensor networks in emergency response: Implementation and pilot results. In Proc. 2008 IEEE Int'l Conf. on Technologies for Homeland Security. Waltham, MA, USA.

Pollack, M., Brown, L., Colbry, D., McCarthy, C., Orosz, C., Peintner, B., Ramakrishnane, S., and Tsamardinos, I. (2003). Autominder: An intelligent cognitive orthotic system for people with memory impairment. Robotics and Autonomous Systems, 44(3-4):273-282. 
Poon, C. Y., Zhang, Y., and Bao, S. (2006). A novel biometrics method to secure wireless body area sensor networks for telemedicine and m-health. IEEE Commun. Mag., 44(4):73-81.

Soomro, A. and Cavalcanti, D. (2007). Opportunities and challenges in using wpan and wlan technologies in medical environments. IEEE Commun. Mag., 45(2):114-122.

Tabar, A., Keshavarz, A., and Aghajan, H. (2006). Smart home care network using sensor fusion and distributed vision-based reasoning. In Proc. 4th ACM Int'l Workshop on Video Surveillance and Sensor Network. Santa Barbara, CA, USA, ACM Press.

Xiao, Y., Shen, X., Sun, B., and Cai, L. (2006). Security and privacy in rfid and applications in telemedicine. IEEE Commun. Mag., 44(4):64-72. 